\title{
Baryogenesis through split Higgsogenesis
}

\author{
Sacha Davidson, ${ }^{a}$ Ricardo González Felipe, ${ }^{b, c}$ Hugo Serôdio ${ }^{d}$ and João P. Silva ${ }^{b, c}$ \\ ${ }^{a} I P N L$, Université de Lyon, Université Lyon 1, CNRS/IN2P3, \\ 4 rue E. Fermi, 69622 Villeurbanne cedex, France \\ ${ }^{b}$ Instituto Superior de Engenharia de Lisboa - ISEL, \\ Rua Conselheiro Emídio Navarro 1, 1959-007 Lisboa, Portugal \\ ${ }^{c}$ Centro de Física Teórica de Partículas (CFTP), \\ Instituto Superior Técnico, Universidade de Lisboa, \\ Avenida Rovisco Pais 1, 1049-001 Lisboa, Portugal \\ ${ }^{d}$ Instituto de Física Corpuscular, Universitat de València - CSIC, \\ Edificio Institutos de Investigación, \\ c/ Catedrático José Beltrán 2, E-46980 Paterna, Valencia, Spain \\ E-mail: s.davidson@ipnl.in2p3.fr, ricardo.felipe@ist.utl.pt, \\ hugo.serodio@ific.uv.es, jpsilva@cftp.ist.utl.pt
}

ABSTRACT: We study the cosmological evolution of asymmetries in the two-Higgs doublet extension of the Standard Model, prior to the electroweak phase transition. If Higgs flavour-exchanging interactions are sufficiently slow, then a relative asymmetry among the Higgs doublets corresponds to an effectively conserved quantum number. Since the magnitude of the Higgs couplings depends on the choice of basis in the Higgs doublet space, we attempt to formulate basis-independent out-of-equilibrium conditions. We show that an initial asymmetry between the Higgs scalars, which could be generated by $\mathrm{CP}$ violation in the Higgs sector, will be transformed into a baryon asymmetry by the sphalerons, without the need of $B-L$ violation. This novel mechanism of baryogenesis through (split) Higgsogenesis is exemplified with simple scenarios based on the out-of-equilibrium decay of heavy singlet scalar fields into the Higgs doublets.

KeYwords: Higgs Physics, CP violation

ARXIV EPRINT: 1307.6218 


\section{Contents}

1 Introduction $\quad 1$

2 The 2 HDM at finite temperature $\quad 2$

2.1 Notation and review 2

2.2 Thermal masses and interaction rates 4

3 Keeping Higgs flavour-exchanging interactions out of equilibrium $\quad 6$

$\begin{array}{lll}3.1 & \text { Basis-independent conditions } & 7\end{array}$

$\begin{array}{llr}4 & \text { Chemical equilibrium relations } & 9\end{array}$

5 Simple split-Higgsogenesis scenarios $\quad \mathbf{1 2}$

$\begin{array}{lll}5.1 & \text { One extra singlet scalar } & 12\end{array}$

$\begin{array}{ll}5.2 \text { One extra singlet and a third doublet } & 14\end{array}$

$\begin{array}{lll}5.3 & \text { Two extra singlet scalars } & 15\end{array}$

$\begin{array}{lll}6 & \text { Summary } & 17\end{array}$

$\begin{array}{ll}\text { A The mass basis and the top basis } & 17\end{array}$

\section{Introduction}

There is at least one Higgs boson [1, 2]; maybe there are more. Multi-Higgs doublet models contain new sources of $\mathrm{CP}$ violation, which is one of the required ingredients [3] for baryogenesis. It is therefore interesting to consider whether $\mathrm{CP}$ violation from the Higgs sector could be used to generate the baryon asymmetry of the Universe [4-7]. This can occur in electroweak baryogenesis scenarios [8-10]; here we are interested in asymmetries produced before the electroweak phase transition (EWPT).

In this paper we consider two-Higgs doublet models (2HDM) [11, 12]. If interactions which exchange Higgs flavour are sufficiently weak, then the two populations of Higgs fields could contain independent asymmetries in the early Universe. Since at least one of the Higgs must couple to Standard Model (SM) fermions, its asymmetry is redistributed among other SM particles by Yukawa interactions, prior to the electroweak phase transition. However, hypercharge neutrality of the Universe relates the asymmetries among all charged particles. This implies that a relative asymmetry among the Higgs scalars, generated by out-ofequilibrium CP-violating processes in the Higgs sector, could be transformed into a baryon asymmetry in the presence of $(B+L)$-violating sphalerons $[13,14]$. The interest of such baryogenesis scenarios is that they require no $B$ or $L$-violating interactions beyond the nonperturbative sphalerons of the SM, relying only on CP violation in an extended Higgs sector. 
The issue of "basis-independence" is of particular importance [15-17]. The point is that physical observables cannot depend on a basis choice in the Lagrangian - one may ask, for instance, what $\phi_{1}$ and $\phi_{2}$ are in the 2HDM. Clearly, the survival of a relative asymmetry between the $\phi_{1}$ 's and $\phi_{2}$ 's in early Universe will depend on the speed of interactions that exchange $\phi_{1}$ with $\phi_{2}$. But the pertinent coupling constants naively appear to depend on the choice of $\phi_{1}$ and $\phi_{2}$. We show that such washout interactions are controlled by the misalignment among different couplings, and can be parameterised in a basis-independent way.

The paper is organized as follows. A compendium of relevant results for the 2HDM is given in section 2.1, followed by some estimates for interaction rates in the early Universe. Section 3 constrains parameters of the Higgs potential by requiring Higgs flavour exchange to be out of equilibrium. In the second part of this section, we discuss the basisindependence of these bounds. In section 4 we derive the equations of chemical equilibrium [18-20], which relate the asymmetries among SM particles and Higgs fields, due to the SM interactions which are in equilibrium, and assuming that the Higgs flavour-exchanging interactions are out of equilibrium. As a result, a nonvanishing equilibrium baryon asymmetry is obtained in the presence of a relative Higgs asymmetry, even with $B-L$ conservation. Simple scenarios based on the out-of-equilibrium decay of singlet scalar fields into Higgs doublets are presented in section 5. Finally, our conclusions are summarized in section 6 .

\section{The $2 \mathrm{HDM}$ at finite temperature}

\section{$2.1 \quad$ Notation and review}

The interaction Lagrangian for the general $2 \mathrm{HDM}[11,12]$ consists of a scalar potential plus Yukawa coupling terms. The most general gauge invariant scalar potential can be written as

$$
\begin{aligned}
V= & m_{11}^{2} \phi_{1}^{\dagger} \phi_{1}+m_{22}^{2} \phi_{2}^{\dagger} \phi_{2}-\left[m_{12}^{2} \phi_{1}^{\dagger} \phi_{2}+\text { h.c. }\right] \\
& +\frac{1}{2} \lambda_{1}\left(\phi_{1}^{\dagger} \phi_{1}\right)^{2}+\frac{1}{2} \lambda_{2}\left(\phi_{2}^{\dagger} \phi_{2}\right)^{2}+\lambda_{3}\left(\phi_{1}^{\dagger} \phi_{1}\right)\left(\phi_{2}^{\dagger} \phi_{2}\right)+\lambda_{4}\left(\phi_{1}^{\dagger} \phi_{2}\right)\left(\phi_{2}^{\dagger} \phi_{1}\right) \\
& +\left[\frac{1}{2} \lambda_{5}\left(\phi_{1}^{\dagger} \phi_{2}\right)^{2}+\lambda_{6}\left(\phi_{1}^{\dagger} \phi_{1}\right)\left(\phi_{1}^{\dagger} \phi_{2}\right)+\lambda_{7}\left(\phi_{2}^{\dagger} \phi_{2}\right)\left(\phi_{1}^{\dagger} \phi_{2}\right)+\text { H.c. }\right],
\end{aligned}
$$

where $\phi_{1}$ and $\phi_{2}$ are two complex $\mathrm{SU}(2)_{L}$ doublet scalar fields of unit hypercharge; $m_{11}^{2}$, $m_{22}^{2}$, and $\lambda_{1} \ldots \lambda_{4}$ are real parameters, while $m_{12}^{2}$ and $\lambda_{5}, \lambda_{6}, \lambda_{7}$ can be complex. In general, both $\phi_{1}$ and $\phi_{2}$ can have Yukawa couplings to all the SM fermions. The Yukawa interactions are

$$
\begin{aligned}
-\mathcal{L}_{Y}= & \overline{Q_{L}}\left(\boldsymbol{\Gamma}_{1} \phi_{1}+\boldsymbol{\Gamma}_{2} \phi_{2}\right) d_{R}+\overline{Q_{L}}\left(\boldsymbol{\Delta}_{1} \tilde{\phi}_{1}+\boldsymbol{\Delta}_{2} \tilde{\phi}_{2}\right) u_{R}+ \\
& +\overline{L_{L}}\left(\boldsymbol{\Pi}_{1} \tilde{\phi}_{1}+\boldsymbol{\Pi}_{2} \tilde{\phi}_{2}\right) \ell_{R}+\text { H.c. }
\end{aligned}
$$

where $Q_{L}=\left(u_{L}, d_{L}\right)^{T}\left(u_{R}\right.$ and $\left.d_{R}\right)$ is a vector in the 3 -dimensional generation space of left-handed quark doublets (right-handed charge $+2 / 3$ and $-1 / 3$ quarks). Accordingly, $\boldsymbol{\Gamma}_{1}$, $\boldsymbol{\Gamma}_{2}, \boldsymbol{\Delta}_{1}$, and $\boldsymbol{\Delta}_{2}$ are $3 \times 3$ matrices in the respective quark generation spaces. Similarly, $L_{L}=\left(\nu_{L}, \ell_{L}\right)^{T}$ and $\ell_{R}$ are vectors in the 3 -dimensional generation space of left-handed lepton doublets and right-handed charged leptons, respectively, while $\boldsymbol{\Pi}_{1}$ and $\boldsymbol{\Pi}_{2}$ are $3 \times 3$ matrices. For simplicity, we assume that there are no right-handed neutrino fields. 


\begin{tabular}{|c|cc|}
\hline Model type & $\phi_{1}$ & $\phi_{2}$ \\
\hline Type I & & $u, d, \ell$ \\
Type II & $d, \ell$ & $u$ \\
Type X & $\ell$ & $u, d$ \\
Type Y & $d$ & $u, \ell$ \\
\hline
\end{tabular}

Table 1. The four types of $Z_{2}$ models and the corresponding Higgs couplings to fermions. Type $\mathrm{X}$ is also known as "lepton specific", and type $\mathrm{Y}$ as "flipped". In the usual $\phi_{u, d}$ notation, $\phi_{u}=\phi_{2}$ always.

Under global $\mathrm{SU}(2)$ transformations in $\left(\phi_{1}, \phi_{2}\right)$ space, the kinetic terms of the Higgs doublets are invariant, whereas the parameters of the scalar potential (and the Yukawa couplings) will be modified. Such basis transformations in the Lagrangian cannot affect observables, so the numerical value of the parameters in eq. (2.1) is only meaningful when the basis is specified. Three obvious Higgs basis choices can be envisaged:

- $m_{12}^{2}=0$ basis, where we put a tilde on the parameters $\left(\tilde{\lambda}_{i}, \tilde{m}_{i i}^{2}, \tilde{y}_{i}^{f}\right)$,

- symmetry basis, where the parameters are lower case with a prime $\left(\lambda_{i}^{\prime}, m_{i j}^{\prime 2}, y_{i}^{\prime f}\right)$,

- (thermal) mass eigenstate basis, where the parameters are uppercase $\left(\Lambda_{i}, M_{i j}, Y_{i}^{f}\right)$.

Here, $\tilde{y}_{i}^{f}, y_{i}^{\prime}{ }_{i}^{f}$ and $Y_{i}^{f}$ denote the Yukawa matrices of the SM fermions $f$ interacting with the Higgs $i$, in the corresponding Higgs basis (so $y_{i}^{u}=\left(\Delta_{1}, \Delta_{2}\right)$, and so on).

Since our goal is to store an asymmetry between the Higgs populations prior to the EWPT, interactions which exchange $\phi_{1} \leftrightarrow \phi_{2}$ must be small (see next section). We refer to such interactions as (Higgs) flavour-exchanging processes. For instance, in the $m_{12}^{2}=0$ basis, the offending parameters from the potential are $\tilde{\lambda}_{5}, \tilde{\lambda}_{6}$ and $\tilde{\lambda}_{7}$. In the Yukawa sector, interactions of both Higgs doublets to either quarks or leptons will be strongly constrained. This is because a relative asymmetry in the two Higgs populations should be preserved, so the two Higgs fields cannot both share their asymmetry with the same fermions.

Some of the undesirable couplings can be suppressed by imposing a discrete $Z_{2}$ symmetry

$$
\phi_{1} \rightarrow-\phi_{1}, \quad \phi_{2} \rightarrow \phi_{2} .
$$

In the basis where the symmetry has the above form, it implies $m_{12}^{\prime}=\lambda_{6}^{\prime}=\lambda_{7}^{\prime}=0$, so the Higgs sector contains no explicit CP violation, because the phase of $\lambda_{5}^{\prime}$ can be rotated away by a phase choice of the Higgs fields. If both scalar fields couple to fermions of the same charge, then there will be flavour changing neutral scalar interactions, which are strongly constrained by experiment. The undesirable Yukawa interactions can be removed by extending the $Z_{2}$ symmetry of eq. (2.3) to the fermion sector, so that each fermion charge sector only couples to one of the Higgs scalars. The four ways to implement this symmetry are shown in table 1.

The discovery of a $125 \mathrm{GeV}$ scalar at the LHC places constraints on the $2 \mathrm{HDM}$ parameter space, studied so far in the context of a $Z_{2}$ symmetry $\left(\lambda_{6}^{\prime}=\lambda_{7}^{\prime}=0\right)$, occasionally 
exact $\left(m_{12}^{\prime}=0\right)$ [21-26]. After electroweak symmetry breaking, the neutral components of the scalar fields acquire the vacuum expectation values (VEVs) $\left\langle\phi_{1}^{0}\right\rangle=v_{1}$ and $\left\langle\phi_{2}^{0}\right\rangle=v_{2}$, where $v=\left(v_{1}^{2}+v_{2}^{2}\right)^{1 / 2} \simeq 174 \mathrm{GeV}$. Of the eight components in the two Higgs doublets, three provide longitudinal components to $W^{ \pm}$and $Z$, two create a $H^{ \pm}$pair, two yield the Higgs scalar $\left(m_{h}=125 \mathrm{GeV}\right)$ and another neutral scalar $(H)$, and the last gives a pseudoscalar $(A)$. These masses and the VEVs are related to the parameters of the potential. In particular, if both $v_{1} \neq 0$ and $v_{2} \neq 0$, then the stationarity conditions can be used to write the pseudoscalar mass

$$
m_{A}^{2}=\frac{v^{2}}{v_{1} v_{2}} m_{12}^{\prime 2}-2 \lambda_{5}^{\prime} v^{2}
$$

This shows that requiring small $\phi_{1} \leftrightarrow \phi_{2}$ exchanges through $m_{12}^{\prime} \sim 0$ and $\lambda_{5}^{\prime} \sim 0$, leads to $m_{A} \sim 0$, unless $v_{1} v_{2} \sim 0$. This occurs because, in the $m_{12}^{\prime}=\lambda_{5}^{\prime}=\lambda_{6}^{\prime}=\lambda_{7}^{\prime}=0$ limit, the potential in eq. (2.1) has a global $\mathrm{U}(1)$ symmetry, which is broken by $v_{1} v_{2} \neq 0$, with the consequent appearance of a massless Goldstone boson $\left(m_{A}=0\right)$.

One solution is to consider the inert model [27-29], which is a Type I 2HDM with exact $Z_{2}$ and $v_{1}=0 .{ }^{1}$ In that case,

$$
m_{A}^{2}=m_{22}^{\prime 2}+\left(\lambda_{3}^{\prime}+\lambda_{4}^{\prime}-\lambda_{5}^{\prime}\right) v^{2}
$$

This mass can be kept nonzero, even if $\lambda_{5}^{\prime}=0$, because the vacuum with $v_{1}=0$ does not break the global U(1). The only consequence of $\lambda_{5}^{\prime}=0$ is $m_{H}=m_{A}$. Because the inert $\phi_{1}$ does not couple to fermions, the lightest particle is a candidate for dark matter. A series of very clear analyses of this model, including constraints from both LHC and WMAP, have been performed by the Warsaw group [30-33]. They find large regions of parameter space consistent with all known data, especially if the $h \rightarrow \gamma \gamma$ signal is consistent with the SM $\left(R_{\gamma \gamma} \sim 1\right)$. This is within the $2 \sigma$ ranges of current ATLAS [34] and CMS [35] data

$$
\begin{aligned}
\text { ATLAS: } & R_{\gamma \gamma}=1.55_{-0.28}^{+0.33}, \\
\text { CMS: } & R_{\gamma \gamma}=0.78_{-0.26}^{+0.28} .
\end{aligned}
$$

Values of $R_{\gamma \gamma}$ larger than one restrict considerably the parameter space.

An additional constraint imposed on the Higgs spectrum by our baryogenesis scenario is that $\phi_{1}$ and $\phi_{2}$ should be present in the thermal bath until the EWPT. If one of the $\phi_{i}$ is sufficiently heavy that its population decays away prior to the EWPT, then the relative Higgs asymmetry is lost.

\subsection{Thermal masses and interaction rates}

In this section we review interaction rates in a thermal bath. The relevant eigenbasis for the external leg particles should be the thermal mass eigenstate basis, so we start by

\footnotetext{
${ }^{1}$ In the usual notation for the inert doublet model, only $\phi_{1}$ couples to fermions, while $v_{2}=0$. In the notation used here, the role of $\phi_{1}$ and $\phi_{2}$ are reversed, implying the changes $m_{11}^{\prime} \leftrightarrow m_{22}^{\prime}$ and $\lambda_{1}^{\prime} \leftrightarrow \lambda_{2}^{\prime}$.
} 
estimating the thermal mass matrix of the Higgs scalars, at temperatures $T \gg\left|m_{i j}\right|$. At finite temperature, the lowest order contribution to the mass-squared matrix is [36]

$$
m_{i j}^{2}(T) \simeq \frac{\partial^{2} V_{\text {eff }}\left(T, \phi_{k}\right)}{\partial \phi_{i} \partial \phi_{j}^{*}}
$$

where $V_{\text {eff }}\left(T, \phi_{k}\right)=V+V_{T}$ is the effective potential, with $V$ given in eq. (2.1). In the high temperature limit $\left(T \gg\left|m_{i j}\right|\right)$,

$$
V_{T}=\frac{T^{2}}{12} \operatorname{Tr}\left[\mathbf{m}^{2}\right]=\frac{T^{2}}{24} \sum_{i=1,2} g_{i} M^{2}\left(\phi_{i}\right),
$$

where $g_{i}=4$ for a complex doublet field, and the trace is calculated over the $T=0$ scalar mass-squared matrix in background field, i.e. allowing $\phi_{1}$ and $\phi_{2}$ to have non-zero values so that $M\left(\phi_{i}\right)$ are field-dependent masses.

Neglecting zero-temperature loop contributions and finite-temperature fermion and gauge contributions, we find

$$
V_{T}=\frac{T^{2}}{12}\left\{3 \lambda_{1}\left|\phi_{1}\right|^{2}+\left(2 \lambda_{3}+\lambda_{4}\right)\left(\left|\phi_{2}\right|^{2}+\left|\phi_{1}\right|^{2}\right)+3 \lambda_{2}\left|\phi_{2}\right|^{2}-\left[3\left(\lambda_{6}+\lambda_{7}\right) \phi_{1}^{\dagger} \phi_{2}+\text { H.c. }\right]\right\},
$$

where the $m_{i j}^{2}$ terms are dropped because they give no contribution to eq. (2.7). For an arbitrary basis in the Higgs doublet space [37, 38], this gives the thermal mass-squared matrix

$$
\mathbf{m}^{2}(T) \simeq \frac{T^{2}}{12}\left(\begin{array}{cc}
3 \lambda_{1}+2 \lambda_{3}+\lambda_{4} & -3 \lambda_{6}-3 \lambda_{7} \\
-3 \lambda_{6}^{*}-3 \lambda_{7}^{*} & 3 \lambda_{2}+2 \lambda_{3}+\lambda_{4}
\end{array}\right)+\left(\begin{array}{cc}
m_{11}^{2} & m_{12}^{2} \\
m_{12}^{* 2} & m_{22}^{2}
\end{array}\right) .
$$

Diagonalising this matrix gives the thermal mass eigenstate basis. In the presence of a $Z_{2}$ symmetry, the thermal masses are simply given by

$$
\begin{aligned}
& m_{11}^{2}(T)=\frac{T^{2}}{12}\left(3 \lambda_{1}+2 \lambda_{3}+\lambda_{4}\right)+m_{11}^{2}, \\
& m_{22}^{2}(T)=\frac{T^{2}}{12}\left(3 \lambda_{2}+2 \lambda_{3}+\lambda_{4}\right)+m_{22}^{2},
\end{aligned}
$$

so that no term of the type $m_{12}^{2}(T) \phi_{1}^{\dagger} \phi_{2}$ is generated. Therefore, in the latter case, the only link between $\phi_{1}$ and $\phi_{2}$ in the Higgs potential comes from the $\lambda_{5}$ term, both at zero and at finite temperature.

We now review the assumptions and approximations involved in our estimates for the interaction rates. We take "thermal equilibrium" to describe a particle species distributed following a Maxwell-Boltzmann distribution. At temperatures $T \ll m_{\mathrm{GUT}} \simeq 10^{16} \mathrm{GeV}$, this will be the case for particles with SM gauge interactions. We define an interaction to be in "chemical equilibrium" if it is fast enough to impose relations among the asymmetries in the participating particles. This will be the case if its timescale, $1 / \Gamma$, is much shorter than the age of the Universe $\sim 1 / H$, i.e. $\Gamma \gg H$, where

$$
H=\sqrt{\frac{4 \pi^{3} g_{*}}{45}} \frac{T^{2}}{m_{P}} \simeq \frac{17 T^{2}}{m_{P}},
$$


is the Hubble expansion parameter, $g_{*}$ is the number of relativistic degrees of freedom $\left(g_{*}=107.75\right.$ in the $\left.2 \mathrm{HDM}\right)$ and $m_{P}=1.22 \times 10^{19} \mathrm{GeV}$ is the Planck mass.

We estimate the interaction rate $\Gamma=\gamma / n$, where $n_{i} \simeq g_{i} T^{3} / \pi^{2}$ is the equilibrium density of an incident (massless) particle, and $\gamma$ is the interaction density. For a process $i j \rightarrow m n$, where all the participating particles are in thermal equilibrium, $\gamma$ is the thermally averaged scattering rate,

$$
\begin{aligned}
\gamma(i j \rightarrow m n) & =\left\langle n_{i} n_{j} \sigma(i+j \rightarrow \ldots)\right\rangle \\
& =\int d \Pi_{i} d \Pi_{j} f_{i}^{e q} f_{j}^{e q} \int|\mathcal{M}(i+j \rightarrow m+n)|^{2}(2 \pi)^{4} \delta^{4}\left(p_{i}+p_{j}-p_{m}-p_{n}\right) d \Pi_{m} d \Pi_{n} \\
& =\frac{g_{1} g_{2}|\Lambda|^{2} T^{4}}{32 \pi^{5}},
\end{aligned}
$$

where $g_{i}$ is the number of degrees of freedom of the particle in the bath (2 for a doublet), $d \Pi=\frac{d^{3} p}{2 E(2 \pi)^{3}}$ is the relativistic phase space, and $f^{e q}$ is the Maxwell-Boltzmann equilibrium distribution. The last equality in eq. (2.13) is the result for $|\mathcal{M}(i+j \rightarrow m+n)|^{2}=|\Lambda|^{2}$.

\section{Keeping Higgs flavour-exchanging interactions out of equilibrium}

We suppose that particle-antiparticle asymmetries in $\phi_{1}$ and $\phi_{2}$ were generated at some earlier epoch of the Universe. In section 5, we shall illustrate this in a simple framework. We focus on the relative asymmetry between the two Higgs doublets:

$$
\mathcal{Y}_{\Delta \phi_{1}}-\mathcal{Y}_{\Delta \phi_{2}} \equiv \frac{n_{\phi_{1}}-n_{\bar{\phi}_{1}}}{s}-\frac{n_{\phi_{2}}-n_{\bar{\phi}_{2}}}{s},
$$

where $s$ is the entropy density of the Universe. We use the notation $\mathcal{Y}_{\Delta X}$ for the asymmetry $\mathcal{Y}_{X}-\mathcal{Y}_{\bar{X}}$, where $\mathcal{Y}_{X}=n_{X} / s$ is the comoving number density. This asymmetry will be conserved as long as Higgs flavour-exchanging interactions are out of equilibrium. In this section, we identify these interactions, estimate the constraints on the couplings, and express these bounds in some useful bases.

In the thermal mass eigenstate basis, the flavour-exchanging Higgs interactions that must be out of equilibrium are mediated by the quartic couplings $\Lambda_{5}, \Lambda_{6}$, and $\Lambda_{7}$. Requiring $\Gamma \ll H$ at $T \simeq 100 \mathrm{GeV}$, and using eqs. (2.12) and (2.13), implies

$$
\left|\Lambda_{n}\right| \lesssim \text { few } \times 10^{-7}, \quad n=5,6,7,
$$

to keep the Higgs asymmetries separate for temperatures down to the EWPT. This condition applies in the thermal mass eigenstate basis; we translate it below to other bases.

Higgs flavour could also be exchanged via Yukawa couplings, if both Higgs doublets interact with the same fermions. For simplicity, we only consider the third generation of fermions. The $t, b$ and $\tau$ have Yukawa interactions to both Higgs fields, so their Yukawa couplings are vectors in Higgs doublet space, which we represent capitalised in the thermal mass eigenstate basis. For instance, the top Yukawa coupling is $\left(Y_{1}^{t}, Y_{2}^{t}\right)$, with $m_{t}=$ $Y_{1}^{t} v_{1}^{T}+Y_{2}^{t} v_{2}^{T}$, where the $v_{i}^{T}$ are the zero-temperature Higgs VEVs in the thermal basis. The 
survival of the relative Higgs asymmetry requires that the Yukawa interactions, between a fermion species $f=t, b, \tau$ and one of the Higgs doublets, be out of equilibrium:

$$
\min _{i} \gamma\left(f+g / \gamma \rightarrow f+\phi_{i}\right) \ll n_{f} H .
$$

For instance, in the case of the top quark, this gives

$$
\theta_{t}^{2} \frac{\alpha_{s}}{16 \pi^{2}} \frac{m_{t}^{2}}{v^{2}} \ll 17 \frac{T}{m_{P}},
$$

where $\theta_{t}$ is the rotation angle between the thermal mass eigenstate Higgs basis and the eigenvector of the top Yukawa coupling (cf. appendix A):

$$
\theta_{t} \simeq \frac{\left|Y_{1}^{t} Y_{2}^{t}\right|}{\sqrt{\left|Y_{1}^{t}\right|^{2}+\left|Y_{2}^{t}\right|^{2}}} \simeq \frac{\left|m_{12}^{2}(T)\right|}{\left|m_{11}^{2}(T)-m_{22}^{2}(T)\right|},
$$

where the last expression is in the Yukawa eigenbasis.

At $T \simeq 100 \mathrm{GeV}$, eq. (3.4) requires

$$
\theta_{t} \lesssim \text { few } \times 10^{-7}
$$

Furthermore, using $\left|\lambda_{6}\right|,\left|\lambda_{7}\right| \lesssim$ few $\times 10^{-7}$ to satisfy eq. (3.2), and assuming $m_{11}^{2}$ and $m_{22}^{2}$ of the order of the lightest Higgs mass, it follows from eqs. (2.10) and (3.5) that

$$
\left|m_{12}^{2}\right| \lesssim(100 \mathrm{MeV})^{2}
$$

in the Yukawa eigenbasis.

We remark that, in obtaining the bound (3.4), we approximate $m_{t}=\left(\left|Y_{1}^{t}\right|^{2}+\left|Y_{2}^{t}\right|^{2}\right)^{1 / 2} v$, that is, we neglect the misalignment between the top Yukawa coupling vector and the zerotemperature VEVs. This could underestimate the magnitude of the Yukawa coupling (as arises for the $b$ and $\tau$ in the large $\tan \beta$ limit of the supersymmetric SM). Therefore, the interaction rates we obtain will be lower bounds. Similar bounds apply to other fermions $f$, with the replacement $m_{t} \rightarrow m_{f}$ (and $\alpha_{s} \rightarrow \alpha_{\mathrm{QED}} / 4$ for leptons). This leads to the bounds

$$
\begin{array}{ll}
\theta_{b} \lesssim 10^{-5} \rightarrow 10^{-7} & \text { for the b quark, } \\
\theta_{\tau} \lesssim 10^{-4} \rightarrow 10^{-6} & \text { for the } \tau \text {-charged lepton }
\end{array}
$$

The weaker limit corresponds to $\left|Y_{1}^{f}\right|^{2}+\left|Y_{2}^{f}\right|^{2}=\left(m_{f} / v\right)^{2}$ and the stronger one to $\left|Y_{1}^{f}\right|^{2}+$ $\left|Y_{2}^{f}\right|^{2} \sim 1$.

\subsection{Basis-independent conditions}

In this section, the conditions given in eqs. (3.2) and (3.4), which ensure the survival of a relative Higgs asymmetry, are expressed in a way which is independent of the (Higgs) basis transformation

$$
\Phi \rightarrow \mathbf{U} \Phi
$$

where $\Phi=\left(\phi_{1}, \phi_{2}\right)^{T}$ and $\mathbf{U}$ is a $2 \times 2$ unitary matrix in Higgs flavour space. In refs. [15-17], basis-independent combinations of potential parameters were constructed by contracting 
the parameters with the Higgs VEVs (a vector in Higgs doublet space). We will construct similar invariants here, but replacing the Higgs VEV with the top Yukawa coupling, which is more relevant for our scenario, and is also a vector in Higgs space (in the one generation approximation). Indeed, one can combine the top Yukawa couplings in

$$
-\mathcal{L}_{Y}=\overline{t_{L}}\left(y_{1}^{t}, y_{2}^{t}\right)\left(\begin{array}{l}
\phi_{1} \\
\phi_{2}
\end{array}\right) t_{R}+\text { h.c. }
$$

into a vector

$$
\hat{y}^{t}=\frac{1}{\sqrt{\left|y_{1}^{t}\right|^{2}+\left|y_{2}^{t}\right|^{2}}}\left(\begin{array}{c}
y_{1}^{t} \\
y_{2}^{t}
\end{array}\right),
$$

transforming as $\hat{y}^{t} \rightarrow \mathbf{U} \hat{y}^{t}$, and its orthogonal

$$
\hat{\epsilon}^{t}=\frac{1}{\sqrt{\left|y_{1}^{t}\right|^{2}+\left|y_{2}^{t}\right|^{2}}}\left(\begin{array}{c}
-y_{2}^{t *} \\
y_{1}^{t *}
\end{array}\right),
$$

transforming as $\hat{\epsilon}^{t} \rightarrow[\operatorname{det} \mathbf{U}]^{-1} \mathbf{U} \hat{\epsilon}^{t}$. In the top basis (see appendix A), these vectors become

$$
\hat{y}^{t}=\left(\begin{array}{l}
1 \\
0
\end{array}\right), \quad \hat{\epsilon}^{t}=\left(\begin{array}{l}
0 \\
1
\end{array}\right) .
$$

From eq. (3.4), it is clear that the direction in Higgs space of the top Yukawa coupling $\hat{y}^{t}$ should approximately correspond to $\phi_{1}$ or $\phi_{2}$ of the thermal mass eigenstate basis. We then simply impose the bounds of eqs. (3.2) and (3.4) in the basis of eq. (3.13).

It is convenient to introduce some notation patterned on ref. [17]. The quartic Higgs interactions can be represented via a four-index tensor which appears in the Lagrangian as $\frac{1}{2} Z_{a \bar{b} c \bar{d}} \Phi_{\bar{a}}^{\dagger} \Phi_{b} \Phi_{\bar{c}}^{\dagger} \Phi_{d}$ where $\Phi^{\dagger}=\left(\phi_{1}^{\dagger}, \phi_{2}^{\dagger}\right)$, and $a, b, c, d=1,2$. The barred (unbarred) notation keeps track of which indices transform as $\mathbf{U}^{\dagger}(\mathbf{U})$, under the basis transformation (3.9). The elements of $Z_{a \bar{b} c \bar{d}}$ are

$$
\begin{array}{ll}
Z_{1 \overline{1} 1 \overline{1}}=\lambda_{1}, & Z_{2 \overline{2} 2 \overline{2}}=\lambda_{2}, \\
Z_{1 \overline{1} 2 \overline{2}}=Z_{2 \overline{2} 1 \overline{1}}=\lambda_{3}, & Z_{1 \overline{2} 2 \overline{1}}=Z_{2 \overline{1} 1 \overline{2}}=\lambda_{4}, \\
Z_{1 \overline{2} 1 \overline{2}}=\lambda_{5}, & Z_{2 \overline{1} 2 \overline{1}}=\lambda_{5}^{*}, \\
Z_{1 \overline{1} 1 \overline{2}}=Z_{1 \overline{1} 1 \overline{1}}=\lambda_{6}, & Z_{1 \overline{1} 2 \overline{1}}=Z_{2 \overline{1} 1 \overline{1}}=\lambda_{6}^{*}, \\
Z_{2 \overline{2} 1 \overline{2}}=Z_{1 \overline{2} 2 \overline{2}}=\lambda_{7}, & Z_{2 \overline{2} 2 \overline{1}}=Z_{2 \overline{1} 2 \overline{2}}=\lambda_{7}^{*} .
\end{array}
$$

By analogy with the invariants $\left|Z_{5}\right|,\left|Z_{6}\right|$, and $\left|Z_{7}\right|$ presented in ref. [17], the following basis invariant quantities can be constructed:

$$
\begin{aligned}
\left|S_{5}\right| & \equiv\left|Z_{a \bar{b} c \bar{d}} \hat{y}_{\bar{a}}^{t *} \hat{\epsilon}_{b}^{t} \hat{y}_{\bar{c}}^{t *} \hat{\epsilon}_{d}^{t}\right|, \\
\left|S_{6}\right| & \equiv\left|Z_{a \bar{b} c \bar{d}} \hat{y}_{\bar{a}}^{t *} \hat{y}_{b}^{t} \hat{y}_{\bar{c}}^{t *} \hat{\epsilon}_{d}^{t}\right|, \\
\left|S_{7}\right| & \equiv\left|Z_{a \bar{b} c \bar{d}} \hat{y}_{\bar{a}}^{*} \bar{\epsilon}_{b}^{t} \hat{\epsilon}_{\bar{c}}^{t *} \hat{\epsilon}_{d}^{t}\right| .
\end{aligned}
$$

These correspond to $\left|\lambda_{5}\right|,\left|\lambda_{6}\right|$, and $\left|\lambda_{7}\right|$ in the basis of eq. (3.13) and, consequently, $\left|S_{n}\right| \lesssim$ few $\times 10^{-7}$ to satisfy eq. (3.2). 
As seen in appendix A, the rotation angle between the thermal mass eigenstate basis and the top Yukawa eigenbasis of eq. (3.13) can be written in the top basis as

$$
\left|\tan \left(2 \theta_{t}\right)\right|=\frac{2\left|m_{12}^{t 2}\right|}{\left|m_{11}^{t 2}-m_{22}^{t 2}\right|}=\frac{2\left|m_{a \bar{b}}^{t 2} \hat{y}_{\bar{a}}^{t *} \hat{\epsilon}_{b}^{t}\right|}{\left|m_{a \bar{b}}^{t 2} \hat{y}_{\bar{a}}^{t *} \hat{y}_{b}^{t}-m_{a \bar{b}}^{t 2} \hat{\epsilon}_{\bar{a}}^{t *} \hat{y}_{b}^{t}\right|},
$$

where the last expression is manifestly basis invariant and, according to eq. (3.6), $\theta_{t} \lesssim$ few $\times 10^{-7}$ to satisfy eq. (3.4).

Finally, the misalignment angles between the top, bottom and tau eigenbases can be formulated in basis-independent notation as

$$
\begin{aligned}
\min \left\{\hat{y}^{b} \cdot \hat{y}^{t}, \hat{y}^{b} \cdot \hat{\epsilon}^{t}\right\} & \simeq \theta_{b} \lesssim 10^{-5} \rightarrow 10^{-7}, \\
\min \left\{\hat{y}^{\tau} \cdot \hat{y}^{t}, \hat{y}^{\tau} \cdot \hat{\epsilon}^{t}\right\} & \simeq \theta_{\tau} \lesssim 10^{-4} \rightarrow 10^{-6},
\end{aligned}
$$

where $\hat{y}^{b}$ and $\hat{y}^{\tau}$ are defined analogously to $\hat{y}^{t}$ in eq. (3.11) and the upper bounds on the right-hand sides follow from eq. (3.8).

In summary, in a 2HDM prior to the EWPT, a relative Higgs asymmetry can survive provided the Yukawa interactions and the Higgs potential have a certain form. The Higgs potential parameters should satisfy the constraints $\left|\lambda_{n}\right| \lesssim$ few $\times 10^{-7}(n=5,6,7)$, and $\left|m_{12}^{2}\right| \lesssim(100 \mathrm{MeV})^{2}$. In the same basis, SM singlet fermions of a given charge (up-type quarks, down-type quarks, charged leptons) should interact with approximately only one Higgs field, that is, the model should be of type I, II, X, or Y.

\section{Chemical equilibrium relations}

Let us now study the redistribution of asymmetries in conserved quantum numbers due to interactions in equilibrium. We assume that Higgs flavour-exchanging interactions are not in equilibrium. We neglect lepton flavour asymmetries, so that the (exactly and effectively) conserved quantum numbers are the hypercharge, $B-L$, and the relative Higgs asymmetry. Assuming that the asymmetries in all species are small, they are related to the chemical potential $\mu$ as

$$
n_{i}-n_{\bar{i}}=\frac{g_{i} T^{2}}{6} \mu_{i} \times\left\{\begin{array}{l}
2 \text { for bosons } \\
1 \text { for fermions }
\end{array},\right.
$$

where $g_{i}$ is the number of degrees of freedom of the particle.

We take the thermal bath to contain the SM fermions and gauge bosons, and two Higgs doublets. We consider temperatures just prior to the EWPT, when all the Yukawa interactions are in equilibrium, but gauge symmetries are unbroken, so that the gauge bosons have zero chemical potential. Our aim is to investigate whether a Higgs asymmetry, as given in eq. (3.1), can be used to generate a baryon asymmetry.

If an interaction is in chemical equilibrium, then the sum of the chemical potentials of the participating particles should vanish. The SM Yukawa interactions impose the relations

$$
-\mu_{q}+\mu_{\phi_{d}}+\mu_{d_{R}}=0
$$




$$
\begin{aligned}
-\mu_{q}-\mu_{\phi_{u}}+\mu_{u_{R}} & =0, \\
-\mu_{\ell}+\mu_{\phi_{e}}+\mu_{e_{R}} & =0,
\end{aligned}
$$

where $\phi_{d}, \phi_{u}$ and $\phi_{e}$ denote the scalar that couples to the down-type quarks, up-type quarks and charged leptons, respectively. Since in the usual notation $\phi_{u}=\phi_{2}$, the various models in table 1 differ by whether $\phi_{d}$ and/or $\phi_{e}$ coincide with $\phi_{2}$.

The electroweak sphalerons impose

$$
3 \mu_{q}+\mu_{\ell}=0,
$$

while the QCD sphalerons lead to the chemical equilibrium condition

$$
2 \mu_{q}-\mu_{u_{R}}-\mu_{d_{R}}=0 .
$$

Adding eqs. (4.2), (4.3), and (4.6), we find

$$
\mu_{\phi_{d}}-\mu_{\phi_{u}}=0
$$

In type II and type Y models, where $\phi_{2}$ couples to up-type quarks, and $\phi_{1}$ to down-type quarks, this forces $\mu_{\phi_{1}}-\mu_{\phi_{2}}$ to vanish [39]. Therefore, in 2HDM of type II and type Y, a relative Higgs asymmetry would be washed out. In contrast, in type I and type X models, $\phi_{d}=\phi_{2}=\phi_{u}$, eq. (4.7) is trivially satisfied, and thus one can have $\mu_{\phi_{1}}-\mu_{\phi_{2}} \neq 0$. Next we show that, provided a Higgs asymmetry was created in early Universe, it can be used to generate a baryon asymmetry at later times.

The baryon and lepton number comoving asymmetries are given by

$$
\begin{aligned}
& \mathcal{Y}_{\Delta B}=N_{g}\left(2 \mu_{q}+\mu_{u_{R}}+\mu_{d_{R}}\right) \frac{T^{2}}{3 s}=4 N_{g} \mu_{q} \frac{T^{2}}{3 s}, \\
& \mathcal{Y}_{\Delta L}=N_{g}\left(2 \mu_{\ell}+\mu_{e_{R}}\right) \frac{T^{2}}{3 s}=-N_{g}\left(9 \mu_{q}+\mu_{\phi_{e}}\right) \frac{T^{2}}{3 s},
\end{aligned}
$$

where $N_{g}=3$ is the number of generations, and eqs. (4.4)-(4.6) have been used to rewrite the right-hand sides of these expressions. As a result,

$$
\mathcal{Y}_{\Delta B}-\mathcal{Y}_{\Delta L}=N_{g}\left(13 \mu_{q}+\mu_{\phi_{e}}\right) \frac{T^{2}}{3 s} .
$$

Finally, hypercharge (or, equivalently, electric) neutrality of the plasma gives

$$
\begin{aligned}
& N_{g}\left(-\mu_{e_{R}}-\mu_{\ell}+\mu_{q}+2 \mu_{u_{R}}-\mu_{d_{R}}\right)+2\left(\mu_{\phi_{1}}+\mu_{\phi_{2}}\right) \\
& =N_{g}\left(8 \mu_{q}+\mu_{\phi_{e}}+3 \mu_{\phi_{2}}\right)+2\left(\mu_{\phi_{1}}+\mu_{\phi_{2}}\right)=0 .
\end{aligned}
$$

The equilibrium baryon asymmetry can then be written as a function of $B-L$ and the Higgs asymmetry:

$$
\begin{array}{ll}
\mathcal{Y}_{\Delta B}=\frac{8}{23}\left(\mathcal{Y}_{\Delta B}-\mathcal{Y}_{\Delta L}\right)+\frac{3}{46}\left(\mathcal{Y}_{\Delta \phi_{1}}-\mathcal{Y}_{\Delta \phi_{2}}\right) & \text { for type I } \\
\mathcal{Y}_{\Delta B}=\frac{8}{23}\left(\mathcal{Y}_{\Delta B}-\mathcal{Y}_{\Delta L}\right)-\frac{33}{92}\left(\mathcal{Y}_{\Delta \phi_{1}}-\mathcal{Y}_{\Delta \phi_{2}}\right) & \text { for type X. }
\end{array}
$$


Eqs. (4.11) and (4.12) give a baryon asymmetry in the presence of a relative Higgs asymmetry, even if $\mathcal{Y}_{\Delta B}-\mathcal{Y}_{\Delta L}=0$. Thus, in these cases, the baryon asymmetry is due exclusively to the initial imbalance between the asymmetry in $\phi_{1}$ and the asymmetry in $\phi_{2}$. We dub this scenario as split Higgsogenesis. As far as we know, this is a novel mechanism for baryogenesis. This is reminiscent of certain asymmetric dark matter (DM) models [40,41], where an asymmetry is generated in a new dark sector (which contains the DM candidate), and then is shared with the SM fermions. The role that the Higgs can play in transferring asymmetries between the SM fermions and the dark sector has been recently emphasized in ref. [42]. However, $\phi_{1}$ does not seem to be a successful asymmetric DM candidate in the simple model discussed here. ${ }^{2}$

Let us assume that, indeed, $\mathcal{Y}_{\Delta B}=\mathcal{Y}_{\Delta L}$. Using eqs. (4.9)-(4.12), we find

$$
\begin{array}{ll}
\mathcal{Y}_{\Delta B}=\frac{3}{46}\left(\mathcal{Y}_{\Delta \phi_{1}}-\mathcal{Y}_{\Delta \phi_{2}}\right)=-\frac{6}{13} \mathcal{Y}_{\Delta \phi_{2}}=\frac{6}{79} \mathcal{Y}_{\Delta \phi_{1}} & \text { for type I, } \\
\mathcal{Y}_{\Delta B}=-\frac{33}{92}\left(\mathcal{Y}_{\Delta \phi_{1}}-\mathcal{Y}_{\Delta \phi_{2}}\right)=-\frac{6}{13} \mathcal{Y}_{\Delta \phi_{1}}=\frac{66}{41} \mathcal{Y}_{\Delta \phi_{2}} & \text { for type X. }
\end{array}
$$

The type I model is particularly interesting because $\phi_{1}$ does not couple to any fermion and could act as dark matter. In that case, $\mathcal{Y}_{\Delta \phi_{1}} / \mathcal{Y}_{\Delta B}=79 / 6$, so the DM scalar should be lighter than the proton ${ }^{3}$ to obtain $\Omega_{\mathrm{DM}} \sim 5 \Omega_{B}$. We recall that the mass density of baryons in the Universe today, as inferred from WMAP in the context of $\Lambda \mathrm{CDM}$ cosmology, is [46]

$$
\frac{m_{p}}{\rho_{c}}\left(n_{B}-n_{\bar{B}}\right)=\Omega_{B} h^{2}=0.02255 \pm 0.0054,
$$

or equivalently,

$$
\mathcal{Y}_{\Delta B}=(8.79 \pm 0.44) \times 10^{-11}
$$

where $m_{p}$ is the proton mass, $h \equiv H_{0} /\left(100 \mathrm{~km} \mathrm{~s}^{-1} \mathrm{Mpc}^{-1}\right)=0.742 \pm 0.036$ is the present Hubble parameter, and $\rho_{c}=3 H_{0}^{2} /(8 \pi G)$ is the critical density of a spatially flat Universe. On the other hand, for the $\Lambda \mathrm{CDM}$ cosmology with three light neutrinos, the cold dark matter relic abundance is $\Omega_{\mathrm{DM}} h^{2}=0.1126 \pm 0.0036$ [46], so that the ratio of dark matter particles to baryons is $\mathcal{Y}_{\mathrm{DM}} / \mathcal{Y}_{\Delta B} \sim 5 m_{p} / m_{\mathrm{DM}}$.

Before concluding this section, it is worth commenting on particular cases of our results, which appeared previously in the literature. In ref. [20], Harvey and Turner have studied a model with $m$ Higgs doublets coupled to all fermions. They evoked mixing between the doublets to maintain equalities of their chemical potentials; for two Higgs, this means $\mathcal{Y}_{\Delta \phi_{2}}=\mathcal{Y}_{\Delta \phi_{1}}$. Using this relation in our eq. (4.11), we recover eq. (9b) of ref. [20] with $N=$ $N_{g}=3$ and $m=2$. In ref. [39], Mohapatra and Zhang pointed out that in a type II 2HDM the equality $\mathcal{Y}_{\Delta \phi_{2}}=\mathcal{Y}_{\Delta \phi_{1}}$ need not be imposed, since it arises from the QCD sphaleron

\footnotetext{
${ }^{2}$ Symmetric dark matter and electroweak baryogenesis have been recently discussed in the inert doublet model in refs. [43-45].

${ }^{3}$ Alternatively, one could assume a primordial $B-L$ asymmetry, no relative Higgs asymmetry, and let the $\lambda_{5}$ coupling (allowed by the $Z_{2}$ symmetry which ensures DM stability) to equilibrate the asymmetry between the two Higgs fields. Then, eq. (4.10) yields a Higgs asymmetry smaller than the baryon asymmetry: $\mathcal{Y}_{\Delta \phi_{1}}=$ $\mathcal{Y}_{\Delta \phi_{2}}=-\mathcal{Y}_{\Delta B} / 8$. This corresponds to a scalar DM mass $\sim 20 \mathrm{GeV}$, which is ruled out by the width of the $Z$ boson. Furthermore, $\lambda_{5}$ would mediate DM-anti-DM oscillations which would wash out the asymmetry.
} 
chemical equilibrium condition, in agreement with our eq. (4.7). Notice however that in these works there is no split Higgsogenesis, and $B-L$ violation is required to generate baryogenesis. We also emphasize that the conditions for the conservation of the relative asymmetry between the Higgs doublets require the flavour-exchanging Higgs interactions to be out of equilibrium. If this requirement is not fulfilled, additional equations for the chemical potentials are introduced, leading to the equality of the Higgs chemical potentials.

\section{$5 \quad$ Simple split-Higgsogenesis scenarios}

Our goal in this section is to provide a few simple scenarios for baryogenesis through splitHiggsogenesis, where the cosmological baryon asymmetry could in principle be generated via the out-of-equilibrium decay of heavy singlet scalars into Higgs doublets.

\subsection{One extra singlet scalar}

We consider an inert 2HDM extended by one real scalar singlet. The two Higgs doublets, $\phi_{1}$ and $\phi_{2}$, and the singlet scalar $S$ transform under $Z_{2}$ as

$$
\phi_{1} \rightarrow-\phi_{1}, \quad \phi_{2} \rightarrow \phi_{2}, \quad S \rightarrow-S
$$

The $Z_{2}$-invariant Higgs potential can be written as

$$
V=V_{\phi}+V_{S}+V_{S \phi}
$$

where

$$
\begin{aligned}
V_{\phi}= & m_{11}^{2}\left|\phi_{1}\right|^{2}+m_{22}^{2}\left|\phi_{2}\right|^{2}+\frac{1}{2} \lambda_{1}\left|\phi_{1}\right|^{4}+\frac{1}{2} \lambda_{2}\left|\phi_{2}\right|^{4}+\lambda_{3}\left|\phi_{1}\right|^{2}\left|\phi_{2}\right|^{2}+\lambda_{4}\left(\phi_{1}^{\dagger} \phi_{2}\right)\left(\phi_{2}^{\dagger} \phi_{1}\right) \\
& +\frac{1}{2} \lambda_{5}\left[\left(\phi_{1}^{\dagger} \phi_{2}\right)^{2}+\text { h.c. }\right] \\
V_{S}= & M^{2} S^{2}+\lambda_{S} S^{4} \\
V_{S \phi}= & z_{1} M\left(\phi_{1}^{\dagger} \phi_{2}\right) S+z_{1}^{*} M\left(\phi_{2}^{\dagger} \phi_{1}\right) S+\beta_{1}\left|\phi_{1}\right|^{2} S^{2}+\beta_{2}\left|\phi_{2}\right|^{2} S^{2} .
\end{aligned}
$$

All parameters are real but $z_{1}$, so that $\mathrm{CP}$ violation in the scalar sector is related with a complex $z_{1}$.

We address now the question whether this model can be used to generate a CP asymmetry in the Higgs sector that could be converted into a baryon asymmetry. The basic idea is analogous to that of the standard leptogenesis scenario. A population of $S$ 's is produced through scattering processes at temperatures $T \sim M \gg m_{\phi_{1}, \phi_{2}}$. This population decays away at $T<M$, when the singlet scalar equilibrium density is Boltzmann suppressed. If the interactions of the heavy singlet $S$ are CP-violating, and provided that the relevant interactions are out of equilibrium, a net Higgs asymmetry can be generated. The latter is then converted into a baryon asymmetry by the sphalerons. To illustrate our mechanism, let us consider the tree-level and one-loop diagrams ${ }^{4}$ depicted in figure 1.

\footnotetext{
${ }^{4}$ There is also a one-loop vertex correction to the tree-level diagram, but since it carries the same phase $z_{1}$, it does not contribute to the Higgs CP asymmetry.
} 

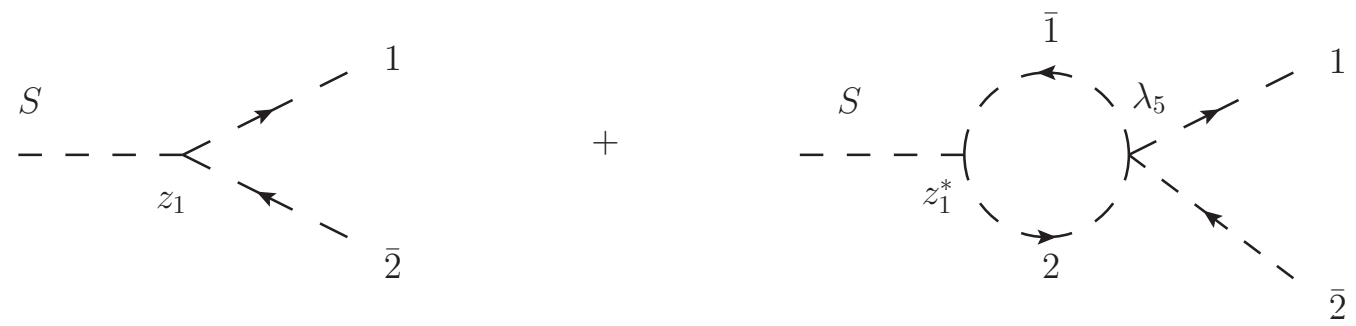

Figure 1. Diagrams contributing to the Higgs CP asymmetry. The notation 1 and $\overline{2}$ refers to $\phi_{1}$ and $\bar{\phi}_{2}$, respectively.

We have two contributions with different CP-odd phases; $z_{1}$ and $z_{1}^{*}$. Because the second diagram is a loop diagram, a cut on this diagram leads to an absorptive part that contains the $\mathrm{CP}$-even phase needed for $\mathrm{CP}$ violation in decays. As a result, the interference of the tree-level and one-loop amplitudes leads to a nonvanishing CP asymmetry in the final Higgs states. Defining this asymmetry as

$$
\epsilon=\frac{\Gamma\left(S \rightarrow \phi_{1} \bar{\phi}_{2}\right)-\Gamma\left(S \rightarrow \bar{\phi}_{1} \phi_{2}\right)}{\Gamma\left(S \rightarrow \phi_{1} \bar{\phi}_{2}\right)+\Gamma\left(S \rightarrow \bar{\phi}_{1} \phi_{2}\right)}
$$

we find

$$
\epsilon \simeq \frac{\overline{\left|c_{0} \mathcal{A}_{0}+c_{1} \mathcal{A}_{1}\right|^{2}}-\overline{\left|c_{0}^{*} \mathcal{A}_{0}+c_{1}^{*} \mathcal{A}_{1}\right|^{2}}}{\mid \overline{c_{0} \mathcal{A}_{0}+\left.c_{1} \mathcal{A}_{1}\right|^{2}}+\overline{\left|c_{0}^{*} \mathcal{A}_{0}+c_{1}^{*} \mathcal{A}_{1}\right|^{2}}} \simeq-4 \frac{\operatorname{Im}\left[c_{0}^{*} c_{1}\right] \operatorname{Im}\left[\overline{\mathcal{A}_{0}^{*} \mathcal{A}_{1}}\right]}{2\left|c_{0}\right|^{2} \mid \overline{\left.\mathcal{A}_{0}\right|^{2}}},
$$

where $\mathcal{A}_{0}$ and $\mathcal{A}_{1}$ are the tree-level and one-loop amplitudes, respectively. For the decay of figure 1 , one has $c_{0}=-z_{1}, c_{1}=3 \lambda_{5} z_{1}^{*}$ and $\mathcal{A}_{0}=1$. Thus, the weak phase gives

$$
\operatorname{Im}\left[c_{0}^{*} c_{1}\right]=-3 \operatorname{Im}\left[z_{1}^{*} \lambda_{5} z_{1}^{*}\right],
$$

while the strong phase comes from

$$
\operatorname{Im}\left[\overline{\left.\mathcal{A}_{0}^{*} \mathcal{A}_{1}\right]}=-\frac{1}{16 \pi} .\right.
$$

We then get

$$
\epsilon \simeq-\frac{3}{8 \pi} \frac{\operatorname{Im}\left[z_{1}^{*} \lambda_{5} z_{1}^{*}\right]}{2\left|z_{1}\right|^{2}}=\frac{3}{16 \pi} \lambda_{5} \sin \left[2 \arg \left(z_{1}\right)\right] .
$$

Thus, in this simple scenario, the Higgs asymmetry is controlled by the strength of the quartic parameter $\lambda_{5}$ and the phase of the coupling $z_{1}$.

The final baryon asymmetry (baryon-to-entropy ratio) can be approximated as

$$
\mathcal{Y}_{\Delta B} \simeq \mathcal{Y}_{S}^{\mathrm{eq}} \times C \times \epsilon \eta,
$$

where the first factor is the equilibrium $S$ number density divided by the entropy density, the second factor is the fraction of the Higgs asymmetry converted into a baryon asymmetry by the sphalerons $(C=3 / 46$ in the present case), and the efficiency factor $\eta(0 \leq \eta \leq 1)$ measures how efficient the out-of-equilibrium $S$-decays are in producing the asymmetry. 
Although a precise computation of $\eta$ requires the solution of a full set of Boltzmann equations, simple analytical estimates can be given. It is useful to introduce the decay parameter

$$
K=\frac{\Gamma_{D}(T=0)}{H(T=M)}
$$

where $\Gamma_{D}=\left|z_{1}\right|^{2} M /(8 \pi)$ is the tree-level decay rate of the singlet $S$ into the two Higgs doublets. In the so-called weak washout regime $(K \ll 1)$, i.e. when the scalar singlet decays strongly out of equilibrium, the efficiency factor is $\eta \simeq 1$. In the strong washout regime $(K \gg 1)$, the efficiency does not depend on the initial conditions, and is mildly suppressed as $\eta \simeq 1 / K$. For intermediate values of $K(K \lesssim 1$ or $K \gtrsim 1)$ the efficiency depends on the assumed initial conditions. We can roughly approximate it as $\eta \sim \min (1,1 / K)$, if $S$ has thermal initial abundance, or $\eta \sim \min (K, 1 / K)$, if $S$ has zero initial abundance.

An estimate for $\mathcal{Y}_{\Delta B}$ can be obtained from eq. (5.9) in the form:

$$
\mathcal{Y}_{\Delta B} \simeq \frac{135 \zeta(3)}{92 \pi^{4} g_{*}} \epsilon \eta \simeq 2 \times 10^{-4} \epsilon \eta .
$$

This is to be compared with the WMAP inferred value given in eq. (4.16). Since eq. (5.8) leads to the upper bound $|\epsilon| \lesssim 6 \times 10^{-2} \lambda_{5}$, then eq. (5.11), when combined with eq. (4.16), requires

$$
\lambda_{5} \eta \gtrsim 7 \times 10^{-6} \text {. }
$$

Notice that between the mass scale $M$ and the EW scale an effective quartic coupling $\lambda_{5}^{\text {eff }}=\lambda_{5}+\frac{1}{2} z_{1}^{2}$ is generated by $S$-exchange. Recalling that, for the relative asymmetry between $\phi_{1}$ and $\phi_{2}$ to survive, interactions which exchange $\phi_{1} \leftrightarrow \phi_{2}$ must be out of equilibrium until the electroweak scale, then eq. (3.2) imposes $\left|\lambda_{5}^{\text {eff }}\right| \lesssim$ few $\times 10^{-7}$. Thus, in this simple setup, unless there is a fine-tuned cancellation between $\lambda_{5}$ and $z_{1}^{2}$ to satisfy this bound, we cannot accommodate the observed baryon asymmetry (4.16), even with a maximal efficiency $\eta \simeq 1$.

\subsection{One extra singlet and a third doublet}

We consider now a model with three doublet scalars and one real scalar singlet. The Higgs doublets, $\phi_{1}, \phi_{2}$ and $\phi_{3}$, and the singlet scalar $S$ transform under $Z_{2}$ as

$$
\phi_{1} \rightarrow-\phi_{1}, \quad \phi_{3} \rightarrow-\phi_{3}, \quad \phi_{2} \rightarrow \phi_{2}, \quad S \rightarrow-S .
$$

The singlet and the third doublet $\phi_{3}$ will be significantly heavier than the EW scale; $\phi_{1}$ and $\phi_{3}$ are defined as the $Z_{2}$-odd mass eigenstates. The $Z_{2}$-invariant Higgs potential can be written as

$$
V_{3}=V+\Delta V_{\phi}+\Delta V_{S \phi}
$$

where $V$ is given in eqs. (5.2)-(5.3) and

$$
\begin{aligned}
\Delta V_{\phi}= & m_{33}^{2}\left|\phi_{3}\right|^{2}+\frac{1}{2} \lambda_{3333}\left|\phi_{3}\right|^{4}+\lambda_{1133}\left|\phi_{1}\right|^{2}\left|\phi_{3}\right|^{2}+\lambda_{2233}\left|\phi_{2}\right|^{2}\left|\phi_{3}\right|^{2} \\
& +\lambda_{1331}\left(\phi_{1}^{\dagger} \phi_{3}\right)\left(\phi_{3}^{\dagger} \phi_{1}\right)+\lambda_{2332}\left(\phi_{2}^{\dagger} \phi_{3}\right)\left(\phi_{3}^{\dagger} \phi_{2}\right)
\end{aligned}
$$




$$
\begin{aligned}
+ & {\left[\frac{1}{2} \lambda_{1313}\left(\phi_{1}^{\dagger} \phi_{3}\right)^{2}+\frac{1}{2} \lambda_{3232}\left(\phi_{3}^{\dagger} \phi_{2}\right)^{2}+\lambda_{1223}\left(\phi_{1}^{\dagger} \phi_{2}\right)\left(\phi_{2}^{\dagger} \phi_{3}\right)+\lambda_{1232}\left(\phi_{1}^{\dagger} \phi_{2}\right)\left(\phi_{3}^{\dagger} \phi_{2}\right)\right.} \\
& \left.+\lambda_{1311}\left(\phi_{1}^{\dagger} \phi_{3}\right)\left(\phi_{1}^{\dagger} \phi_{1}\right)+\lambda_{1322}\left(\phi_{1}^{\dagger} \phi_{3}\right)\left(\phi_{2}^{\dagger} \phi_{2}\right)+\lambda_{1333}\left(\phi_{1}^{\dagger} \phi_{3}\right)\left(\phi_{3}^{\dagger} \phi_{3}\right)+\text { H.c. }\right], \\
\Delta V_{S \phi}= & z_{3} M\left(\phi_{3}^{\dagger} \phi_{2}\right) S+z_{3}^{*} M\left(\phi_{2}^{\dagger} \phi_{3}\right) S+\beta_{3}\left|\phi_{3}\right|^{2} S^{2}+\left[\beta_{13} \phi_{1}^{\dagger} \phi_{3} S^{2}+\text { H.c. }\right] .
\end{aligned}
$$

The basic idea is similar to the $2 \mathrm{HDM}$ with an extra singlet. A population of $S$ 's is produced through scattering processes at temperatures $T \sim M>m_{33} \gg m_{\phi_{1}, \phi_{2}}$. This population decays away at $T<M$, when the singlet scalar equilibrium density is Boltzmann suppressed. If the interactions of the heavy singlet $S$ are CP-violating, asymmetries among the three Higgs doublets can be generated. When washout interactions are out of equilibrium, the asymmetries can survive. The $\phi_{3}$ later decay to $\phi_{1}$, leaving an asymmetry between $\phi_{2}$ and $\phi_{1}$. Assuming the asymmetry from $S \rightarrow \phi_{1} \phi_{2}^{*}$ to be negligible, due to the bounds on $\lambda_{5}$ and $z_{1}^{2}$, we neglect it and focus on a possible asymmetry from $S \rightarrow \phi_{3} \phi_{2}^{*}$.

We consider tree-level and one-loop diagrams analogous to those depicted in figure 1 , with $\phi_{1} \rightarrow \phi_{3}, z_{1} \rightarrow z_{3}$, and $\lambda_{5} \rightarrow \lambda_{3232}$. The interference of the tree-level and one-loop amplitudes leads to a nonvanishing CP asymmetry in the final Higgs states:

$$
\epsilon=\frac{\Gamma\left(S \rightarrow \phi_{3} \bar{\phi}_{2}\right)-\Gamma\left(S \rightarrow \bar{\phi}_{3} \phi_{2}\right)}{\Gamma(S \rightarrow \text { all })} \simeq-\frac{3}{8 \pi} \frac{\operatorname{Im}\left[z_{3}^{*} \lambda_{3232} z_{3}^{*}\right]}{2\left|z_{3}\right|^{2}}=\frac{3}{16 \pi} \lambda_{3232} \sin \left[2 \arg \left(z_{3}\right)\right],
$$

where the contribution of $z_{1}$ to the total decay rate has been neglected.

For the relative asymmetry between $\phi_{3}+\phi_{1}$ and $\phi_{2}$ to survive, interactions which exchange $\phi_{3}$ with $\phi_{2}$ must be out of equilibrium until the $\phi_{3}$ decay. (Recall that we have already imposed that interactions exchanging $\phi_{1} \leftrightarrow \phi_{2}$ are out of thermal equilibrium). By extrapolating eq. (3.2) to higher temperatures, this imposes

$$
\left|z_{3}\right|^{2},\left|\lambda_{3232}\right|<\text { few } \times 10^{-7} \sqrt{\frac{m_{33}}{\mathrm{TeV}}},
$$

assuming that $S$-exchange generates an effective $\lambda_{3232}^{\text {eff }}=\lambda_{3232}+\frac{1}{2} z_{3}^{2}$ between the temperature scales $M$ and $m_{33}$, and that $\phi_{3}$ decay at $T \simeq 10 m_{33}$. From eqs. (5.11) and (4.16), a large enough asymmetry is obtained for $m_{33} \gtrsim 10^{8} \mathrm{GeV}$, provided the washout effects are not too strong, $\eta \simeq \mathcal{O}(0.1)$. Since the efficiency of Higgsogenesis is controlled by the decay parameter $K$ defined in eq. (5.10),

$$
K=\frac{\left|z_{3}\right|^{2}}{136 \pi} \frac{m_{P}}{M} \simeq 3 \times\left(\frac{\left|z_{3}\right|^{2}}{10^{-4}}\right) \times\left(\frac{10^{12} \mathrm{GeV}}{M}\right),
$$

this implies $M \gtrsim 10^{12} \mathrm{GeV}$.

\subsection{Two extra singlet scalars}

Let us now consider a model with two real scalar singlets $S_{i}(i=1,2)$, both transforming under $Z_{2}$ as $S_{i} \rightarrow-S_{i}$. The Higgs potential can be written as in eqs. (5.2)-(5.3), but in this case $V_{S}$ and $V_{S \phi}$ contain additional terms. In particular, $V_{S \phi}$ contains the cubic 

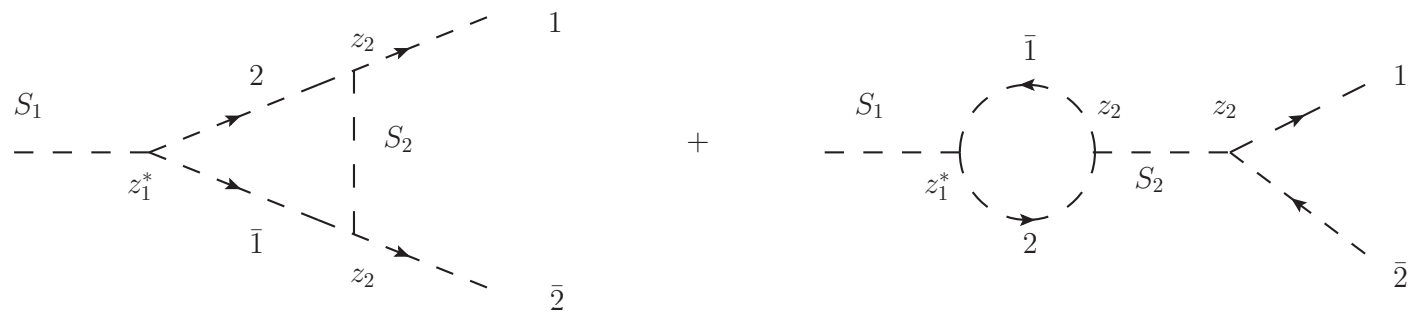

Figure 2. New diagrams contributing to the Higgs CP asymmetry in the presence of two singlet scalars. The notation 1 and $\overline{2}$ refers to $\phi_{1}$ and $\bar{\phi}_{2}$, respectively.

terms $z_{i} M_{i}\left(\phi_{1}^{\dagger} \phi_{2}\right) S_{i}+z_{i}^{*} M_{i}\left(\phi_{2}^{\dagger} \phi_{1}\right) S_{i}$, in addition to six quartic terms. Similarly, $V_{S}$ has several quartic terms and, without loss of generality, we choose a $\left\{S_{1}, S_{2}\right\}$ basis where the quadratic terms are already diagonalized, and assume $M_{1}<M_{2}$.

In what follows, we make the following simplifying assumptions: the heavy singlet spectrum is hierarchical, $M_{1} \ll M_{2}$; there is a thermal production of $S_{1}$ and negligible production of $S_{2}$. With these assumptions, the Higgsogenesis mechanism will proceed via the out-of-equilibrium decays of $S_{1}$. The decay $S_{1} \rightarrow \phi_{1} \bar{\phi}_{2}$ is still mediated by the diagrams in figure 1, but we also have the additional (vertex and self-energy) diagrams depicted in figure 2 .

These diagrams carry the phase of $z_{1}^{*} z_{2}^{2}$, which will be beaten against the $z_{1}$ phase from the tree level diagram in figure 1 . As a result, in this model, even if $\lambda_{5}$ vanishes, there is a CP-violating contribution proportional to $\operatorname{Im}\left(z_{1}^{* 2} z_{2}^{2}\right)$. The resulting $\mathrm{CP}$ asymmetry, as given in eq. (5.4), can be evaluated following the standard procedure. Neglecting the $\lambda_{5}$ contribution coming from the one-loop diagram of figure 1, we obtain

$$
\epsilon_{1} \simeq-\frac{1}{4 \pi} \frac{\operatorname{Im}\left[\left(z_{1}^{*} z_{2}\right)^{2}\right]}{\left|z_{1}\right|^{2}}\left[\frac{1}{2} f\left(x_{2}\right)+g\left(x_{2}\right)\right],
$$

where $x_{2}=M_{2}^{2} / M_{1}^{2}$, and

$$
f(x)=x \ln \left(\frac{x}{1+x}\right), \quad g(x)=\frac{x}{1-x},
$$

are the vertex and self-energy one-loop functions, respectively.

In the hierarchical limit, $x_{2} \gg 1, f\left(x_{2}\right) \simeq-1, g\left(x_{2}\right) \simeq-1$, and the CP asymmetry is approximately given by

$$
\epsilon_{1} \simeq \frac{3}{8 \pi} \frac{\operatorname{Im}\left[\left(z_{1}^{*} z_{2}\right)^{2}\right]}{\left|z_{1}\right|^{2}}=\frac{3}{8 \pi}\left|z_{2}\right|^{2} \sin \left[2 \arg \left(z_{1}^{*} z_{2}\right)\right] .
$$

From eqs. (5.11), (4.16) and (5.21) we then conclude that a successful generation of the baryon asymmetry within the present model requires

$$
\left|z_{2}\right|^{2} \eta \gtrsim \text { few } \times 10^{-6}
$$

which in turn implies that $\left|z_{2}\right|^{2} \gtrsim$ few $\times 10^{-6}$. Yet, as in the case with one extra singlet, an effective quartic coupling $\lambda_{5}^{\text {eff }}=\lambda_{5}+\frac{1}{2} z_{1}^{2}+\frac{1}{2} z_{2}^{2}$ is generated by $S$-exchange between the $M$ 
and EW temperature scales. The latter should satisfy the bound $\left|\lambda_{5}^{\text {eff }}\right| \lesssim$ few $\times 10^{-7}$. So, in this case, to accommodate the observed baryon asymmetry (4.16) we should have some relation between $\lambda_{5}, z_{1}^{2}$ and/or $z_{2}^{2}$; for example, $z_{1} \simeq i z_{2}$ to avoid the restrictive bounds on these couplings.

\section{Summary}

In this work, we have studied the possibility of generating the cosmological baryon asymmetry in the context of 2HDM extensions of the SM, prior to the electroweak phase transition. We have shown that if the Higgs-flavour exchanging interactions are sufficiently slow in the early Universe, then a relative asymmetry among the Higgs doublets corresponds to an effectively conserved quantum number. Such a relative Higgs asymmetry can be transformed into a baryon asymmetry by the sphalerons, without the need for $B-L$ violation.

Among the four possible types of $Z_{2}$ models considered, we have demonstrated that this "split Higgsogenesis" mechanism is only possible in the framework of a type-I or type-X 2HDM. We then presented simple scenarios to generate a Higgs asymmetry, based on inert type-I 2HDMs extended by heavy singlet scalar fields and/or one extra Higgs doublet. In the presence of CP-violating interactions, the out-of-equilibrium decays of the heavy singlets into the Higgs doublets can produce a net Higgs asymmetry and the mechanism of baryogenesis through (split) Higgsogenesis can be viable. Since a successful implementation of our mechanism requires the scalar potential parameters to satisfy definite bounds, we have also paid particular attention to their basis-independent formulation.

\section{Acknowledgments}

We are grateful to A. Barroso, M. Krawczyk and R. Santos for useful discussions. S.D. acknowledges partial support from the EU FP7 ITN INVISIBLES (MC actions, PITNGA-2011-289-442) and the Lyon Institute of Origins. The work of R.G.F. and J.P.S. was partially supported by FCT - Fundação para a Ciência e a Tecnologia, under the Projects PEst-OE/FIS/UI0777/2011 and CERN/FP/123580/2011, and by the EU RTN Marie Curie Project PITN-GA-2009-237920. The work of H.S. is funded by the European FEDER and Spanish MINECO, under the Grant FPA2011-23596.

\section{A The mass basis and the top basis}

Let us consider the quadratic terms of the scalar potential,

$$
V_{2}=\left(\begin{array}{ll}
\phi_{1}^{\dagger} & \phi_{2}^{\dagger}
\end{array}\right) \mathbf{M}\left(\begin{array}{l}
\phi_{1} \\
\phi_{2}
\end{array}\right),
$$

where

$$
\mathbf{M}=\left(\begin{array}{cc}
m_{11}^{2} & -m_{12}^{2} \\
-\left(m_{12}^{2}\right)^{*} & m_{22}^{2}
\end{array}\right)
$$


is a Hermitian matrix. Its eigenvalues are

$$
M_{1,2}^{2}=\frac{m_{11}^{2}+m_{22}^{2}}{2} \pm \frac{1}{2} \sqrt{\left(m_{11}^{2}-m_{22}^{2}\right)^{2}+4\left|m_{12}^{2}\right|^{2}} .
$$

The top Yukawa couplings can be written as

$$
-\mathcal{L}_{Y}=\bar{t}_{L}\left(\begin{array}{ll}
y_{1}^{t} & y_{2}^{t}
\end{array}\right)\left(\begin{array}{c}
\tilde{\phi}_{1} \\
\tilde{\phi}_{2}
\end{array}\right) t_{R}+\text { H.c. },
$$

from which we may build the Hermitian matrix

$$
\mathbf{H}=\left(\begin{array}{cc}
\left|y_{1}^{t}\right|^{2} & y_{1}^{t} y_{2}^{t *} \\
y_{1}^{t *} y_{2}^{t} & \left|y_{2}^{t}\right|^{2}
\end{array}\right) .
$$

Its eigenvalues are

$$
\left(y^{\text {top }}\right)^{2}=\left|y_{1}^{t}\right|^{2}+\left|y_{2}^{t}\right|^{2}
$$

and zero.

In the mass basis, $\mathbf{M}$ is diagonal and $\mathbf{H}$ has the form

$$
\mathbf{H}=\left(\begin{array}{cc}
\left|y_{1}^{t \mathrm{~m}}\right|^{2} & y_{1}^{t \mathrm{~m}} y_{2}^{t \mathrm{~m} *} \\
y_{1}^{t \mathrm{~m} *} y_{2}^{t \mathrm{~m}} & \left|y_{2}^{t \mathrm{~m}}\right|^{2}
\end{array}\right) .
$$

In the top basis, $\mathbf{H}$ is diagonal, only one Higgs scalar couples to the top, and

$$
\mathbf{M}=\left(\begin{array}{cc}
m_{11}^{\mathrm{t} 2} & -m_{12}^{\mathrm{t} 2} \\
-\left(m_{12}^{\mathrm{t} 2}\right)^{*} & m_{22}^{\mathrm{t} 2}
\end{array}\right)
$$

Notice that we have used the superscript " $\mathrm{m}$ " to stress that the matrix elements of $\mathbf{H}$ are to be calculated in the basis where $\mathbf{M}$ is diagonal. Similarly, the superscript "t" indicates that the matrix elements of $\mathbf{M}$ are to be calculated in the basis where $\mathbf{H}$ is diagonal.

There is physical content in the misalignment between the two bases, which can be expressed in a basis-invariant way through the relation

$$
\sin ^{2}\left(2 \theta_{t}\right)=\frac{4 \operatorname{det}[\mathbf{H}, \mathbf{M}]}{\left[2 \operatorname{Tr}\left(\mathbf{H}^{2}\right)-(\operatorname{Tr} \mathbf{H})^{2}\right]\left[2 \operatorname{Tr}\left(\mathbf{M}^{2}\right)-(\operatorname{Tr} \mathbf{M})^{2}\right]} .
$$

In the top basis, the above equation yields

$$
\sin ^{2}\left(2 \theta_{t}\right)=\frac{4\left|m_{12}^{\mathrm{t} 2}\right|^{2}}{\left(m_{11}^{\mathrm{t} 2}-m_{22}^{\mathrm{t} 2}\right)^{2}+4\left|m_{12}^{\mathrm{t} 2}\right|^{2}},
$$

or equivalently,

$$
\left|\tan \left(2 \theta_{t}\right)\right|=\frac{2\left|m_{12}^{\mathrm{t} 2}\right|}{\left|m_{11}^{\mathrm{t} 2}-m_{22}^{\mathrm{t} 2}\right|}
$$

In the mass basis, it leads to

$$
\sin ^{2}\left(2 \theta_{t}\right)=\frac{4\left|y_{1}^{t \mathrm{~m}}\right|^{2}\left|y_{2}^{t \mathrm{~m}}\right|^{2}}{\left|y_{1}^{t \mathrm{~m}}\right|^{2}+\left|y_{2}^{t \mathrm{~m}}\right|^{2}}
$$


For small angles we can then write

$$
\theta_{t} \simeq \frac{\left|y_{1}^{t \mathrm{~m}} y_{2}^{t \mathrm{~m}}\right|}{\sqrt{\left|y_{1}^{t \mathrm{~m}}\right|^{2}+\left|y_{2}^{t \mathrm{~m}}\right|^{2}}} \simeq \frac{\left|m_{12}^{\mathrm{t} 2}\right|}{\left|m_{11}^{\mathrm{t} 2}-m_{22}^{\mathrm{t} 2}\right|}
$$

This equation provides two different ways of writing the misalignment between the top and the mass bases to be used in the text.

Thus far we did not need to specify whether the mass matrix $\mathbf{M}$ in eq. (A.2) is to be calculated at a finite temperature $T$, or at $T=0$; the expressions hold for any case. But one subtlety must be pointed out when using eq. (A.13) with a temperature-dependent matrix $\mathbf{M}(T)$. The thermal mass basis rotates as the temperature varies, as can be seen from eq. (2.10). The top basis, on the other hand, is temperature independent, and the bound of eq. (3.4) must be satisfied from the temperature when the Higgs asymmetry is created until the EWPT. This implies that the thermal basis cannot rotate much during this period.

Open Access. This article is distributed under the terms of the Creative Commons Attribution License which permits any use, distribution and reproduction in any medium, provided the original author(s) and source are credited.

\section{References}

[1] CMS collaboration, Observation of a new boson at a mass of $125 \mathrm{GeV}$ with the CMS experiment at the LHC, Phys. Lett. B 716 (2012) 30 [arXiv:1207.7235] [INSPIRE].

[2] ATLAS collaboration, Observation of a new particle in the search for the Standard Model Higgs boson with the ATLAS detector at the LHC, Phys. Lett. B 716 (2012) 1 [arXiv: 1207.7214] [INSPIRE].

[3] A. Sakharov, Violation of CP invariance, $c$ asymmetry and baryon asymmetry of the universe, Pisma Zh. Eksp. Teor. Fiz. 5 (1967) 32 [JETP Lett. 5 (1967) 24] [Sov. Phys. Usp. 34 (1991) 392] [Usp. Fiz. Nauk 161 (1991) 61] [INSPIRE].

[4] E.W. Kolb and S. Wolfram, Baryon number generation in the early universe, Nucl. Phys. B 172 (1980) 224 [Erratum ibid. B 195 (1982) 542] [InSPIRE].

[5] A. Dolgov, Non-GUT baryogenesis, Phys. Rept. 222 (1992) 309 [INSPIRE].

[6] For recent reviews see e.g. S. Davidson, E. Nardi and Y. Nir, Leptogenesis, Phys. Rept. 466 (2008) 105 [arXiv: 0802.2962] [INSPIRE].

[7] G. Branco, R.G. Felipe and F. Joaquim, Leptonic CP-violation, Rev. Mod. Phys. 84 (2012) 515 [arXiv:1111.5332] [InSPIRE].

[8] V. Rubakov and M. Shaposhnikov, Electroweak baryon number nonconservation in the early universe and in high-energy collisions, Usp. Fiz. Nauk 166 (1996) 493

[Phys. Usp. 39 (1996) 461] [hep-ph/9603208] [INSPIRE].

[9] M. Quirós, Electroweak baryogenesis, J. Phys. A 40 (2007) 6573 [INSPIRE].

[10] J.M. Cline, Baryogenesis, hep-ph/0609145 [INSPIRE].

[11] J.F. Gunion, H.E. Haber, G. Kane and S. Dawson, The Higgs hunter's guide, Perseus Publishing, Cambridge MA U.S.A. (1990). 
[12] For a recent review on 2HDM see for example G. Branco et al., Theory and phenomenology of two-Higgs-doublet models, Phys. Rept. 516 (2012) 1 [arXiv:1106.0034] [INSPIRE].

[13] P.B. Arnold and L.D. McLerran, Sphalerons, small fluctuations and baryon number violation in electroweak theory, Phys. Rev. D 36 (1987) 581 [inSPIRE].

[14] F.R. Klinkhamer and N. Manton, A saddle point solution in the Weinberg-Salam theory, Phys. Rev. D 30 (1984) 2212 [INSPIRE].

[15] L. Lavoura and J.P. Silva, Fundamental CP-violating quantities in a $\mathrm{SU}(2) \times \mathrm{U}(1)$ model with many Higgs doublets, Phys. Rev. D 50 (1994) 4619 [hep-ph/9404276] [INSPIRE].

[16] F. Botella and J.P. Silva, Jarlskog-like invariants for theories with scalars and fermions, Phys. Rev. D 51 (1995) 3870 [hep-ph/9411288] [INSPIRE].

[17] S. Davidson and H.E. Haber, Basis-independent methods for the two-Higgs-doublet model, Phys. Rev. D 72 (2005) 035004 [Erratum ibid. D 72 (2005) 099902] [hep-ph/0504050] [INSPIRE].

[18] S.Y. Khlebnikov and M. Shaposhnikov, The statistical theory of anomalous Fermion number nonconservation, Nucl. Phys. B 308 (1988) 885 [INSPIRE].

[19] S.Y. Khlebnikov and M. Shaposhnikov, Melting of the Higgs vacuum: conserved numbers at high temperature, Phys. Lett. B 387 (1996) 817 [hep-ph/9607386] [INSPIRE].

[20] J.A. Harvey and M.S. Turner, Cosmological baryon and lepton number in the presence of electroweak fermion number violation, Phys. Rev. D 42 (1990) 3344 [INSPIRE].

[21] For recent reviews see for example B. Grinstein and P. Uttayarat, Carving out parameter space in type-II two Higgs doublets model, JHEP 06 (2013) 094 [Erratum ibid. 09 (2013) 110] [arXiv: 1304.0028] [INSPIRE].

[22] A. Barroso, P. Ferreira, R. Santos, M. Sher and J.P. Silva, 2HDM at the LHC - the story so far, arXiv:1304.5225 [inSPIRE].

[23] C.-Y. Chen, S. Dawson and M. Sher, Heavy Higgs searches and constraints on two Higgs doublet models, Phys. Rev. D 88 (2013) 015018 [arXiv: 1305.1624] [INSPIRE].

[24] O. Eberhardt, U. Nierste and M. Wiebusch, Status of the two-Higgs-doublet model of type-II, arXiv: 1305.1649 [INSPIRE].

[25] N. Craig, J. Galloway and S. Thomas, Searching for signs of the second Higgs doublet, arXiv:1305. 2424 [INSPIRE].

[26] P. Ferreira, R. Santos, M. Sher and J.P. Silva, 2HDM confronting LHC data, arXiv: 1305.4587 [INSPIRE].

[27] N.G. Deshpande and E. Ma, Pattern of symmetry breaking with two Higgs doublets, Phys. Rev. D 18 (1978) 2574 [inSPIRE].

[28] R. Barbieri, L.J. Hall and V.S. Rychkov, Improved naturalness with a heavy Higgs: an alternative road to LHC physics, Phys. Rev. D 74 (2006) 015007 [hep-ph/0603188] [INSPIRE].

[29] Q.-H. Cao, E. Ma and G. Rajasekaran, Observing the dark scalar doublet and its impact on the Standard Model Higgs boson at colliders, Phys. Rev. D 76 (2007) 095011 [arXiv: 0708.2939] [INSPIRE].

[30] M. Krawczyk, D. Sokolowska and B. Swiezewska, Inert doublet model with a $125 \mathrm{GeV}$ Higgs, arXiv: 1304.7757 [INSPIRE]. 
[31] M. Krawczyk, D. Sokolowska, P. Swaczyna and B. Swiezewska, Constraining inert dark matter by $R_{\gamma \gamma}$ and WMAP data, JHEP 09 (2013) 055 [arXiv:1305.6266] [INSPIRE].

[32] B. Swiezewska and M. Krawczyk, 2-photon decay rate of the scalar boson in the inert doublet model, arXiv:1305.7356 [INSPIRE].

[33] B. Swiezewska, Yukawa independent constraints for 2 HDMs with a $125 \mathrm{GeV}$ Higgs boson, Phys. Rev. D 88 (2013) 055027 [arXiv: 1209.5725] [INSPIRE].

[34] ATLAS collaboration, Measurements of Higgs boson production and couplings in diboson final states with the ATLAS detector at the LHC, Phys. Lett. B 726 (2013) 88 [arXiv: 1307.1427] [INSPIRE].

[35] CMS collaboration, Updated measurements of the Higgs boson at $125 \mathrm{GeV}$ in the two photon decay channel, CMS-PAS-HIG-13-001, CERN, Geneva Switzerland (2013).

[36] D. Comelli and J. Espinosa, Bosonic thermal masses in supersymmetry, Phys. Rev. D 55 (1997) 6253 [hep-ph/9606438] [INSPIRE].

[37] I. Ivanov, Thermal evolution of the ground state of the most general 2HDM, Acta Phys. Polon. B 40 (2009) 2789 [arXiv:0812.4984] [INSPIRE].

[38] I. Ginzburg, I. Ivanov and K. Kanishev, The evolution of vacuum states and phase transitions in 2HDM during cooling of universe, Phys. Rev. D 81 (2010) 085031 [arXiv: 0911.2383] [INSPIRE].

[39] R.N. Mohapatra and X.-M. Zhang, QCD sphalerons at high temperature and baryogenesis at electroweak scale, Phys. Rev. D 45 (1992) 2699 [INSPIRE].

[40] H. Davoudiasl and R.N. Mohapatra, On relating the genesis of cosmic baryons and dark matter, New J. Phys. 14 (2012) 095011 [arXiv:1203.1247] [INSPIRE].

[41] K. Petraki and R.R. Volkas, Review of asymmetric dark matter, Int. J. Mod. Phys. A 28 (2013) 1330028 [arXiv:1305.4939] [INSPIRE].

[42] G. Servant and S. Tulin, Higgsogenesis, Phys. Rev. Lett. 111 (2013) 151601 [arXiv: 1304.3464] [INSPIRE].

[43] D. Borah and J.M. Cline, Inert doublet dark matter with strong electroweak phase transition, Phys. Rev. D 86 (2012) 055001 [arXiv:1204.4722] [INSPIRE].

[44] G. Gil, P. Chankowski and M. Krawczyk, Inert dark matter and strong electroweak phase transition, Phys. Lett. B 717 (2012) 396 [arXiv:1207.0084] [INSPIRE].

[45] M. Laine, G. Nardini and K. Rummukainen, Lattice study of an electroweak phase transition at $m_{h} \sim 126 \mathrm{GeV}, J C A P 01$ (2013) 011 [arXiv:1211.7344] [INSPIRE].

[46] WMAP collaboration, E. Komatsu et al., Seven-year Wilkinson Microwave Anisotropy Probe (WMAP) observations: cosmological interpretation, Astrophys. J. Suppl. 192 (2011) 18 [arXiv:1001.4538] [INSPIRE]. 